\title{
ESTRUCTURAS DE EMPLEO DEL TIEMPO EN LA COMUNIDAD DE CASTILLA-LA MANCHA
}

\section{Javier Callejo*}

\section{RESUMEN}

A partir de los resultados de la Encuesta de Empleo del Tiempo, operación estadística desarrollada por el Instituto Nacional de Estadística durante 2002 y 2003, se ofrece una panorámica del empleo social medio que hace la sociedad de Castilla-La Mancha del tiempo. Además de a las categorías que sirven para agrupar el complejo tejido de actividades que los sujetos realizan a lo largo de una jornada, se ha dado especial relevancia a las siguientes: evolución del tiempo dedicado a las distintas actividades a lo largo de la semana, tamaño de localidad y, por último y con la intención de abordar también aspectos cualitativos de la vivencia del tiempo, el sentimiento de agobio.

\section{PALABRAS CLAVE}

Presupuestos temporales, sociología del tiempo, tamaño de localidad, sentimiento de agobio.

\begin{abstract}
From the results of the Survey of Use of Time, statistical operation developed by the National Institute of Statistics during 2002 and 2003, a pan of the social use of time of Castilla-La Mancha is offered. Besides from to the categories that serve to group the complex woven of activities that the subjects carry out along a day, special importance to the following has been given: evolution of the amount of time dedicated to the different activities along the week, size of locality and, finally and with the intention to undertake also qualitative aspects of the experience of the time, the feeling of burden.
\end{abstract}

\section{KEY WORDS}

Time budgets, sociology of time, size of locality, feeling of burden

\section{PRESENTACIÓN}

Los estudios sobre el uso del tiempo se han asentado en la mayor parte de los países desarrollados, con un horizonte internacional claramente destinado a la comparación entre unos países y otros ${ }^{1}$. Tal interés por el estudio de cómo las sociedades usan el tiempo deriva principalmente de su consideración como un recurso, relativamente igual para todas; pero que es susceptible de ser aplicado y distribuido de muy distinta manera según cada sociedad. Visto así, el uso del tiempo

\footnotetext{
*Profesor titular en la Facultad de Ciencias Políticas y Sociología. Universidad Nacional de Educación a Distancia
} 
se convierte en una especie de privilegiado indicador de la sociedad, capaz de condensarla en buena medida: habla de la sociedad. Usando una socorrida metáfora: el uso del tiempo se configura como retrato de la sociedad.

Ha de reconocerse que la mayor parte del esfuerzo institucional por saber el uso del tiempo en las sociedades se ha concentrado en la perspectiva cuantitativa, mediante encuesta con cuestionario estandarizado, llegándose a cierta homogeneización de las técnicas de estudio de empleo del tiempo (Szalai, 1972). Así se ha configurado un instrumento que ha adquirido nombre propio en el ámbito de los métodos y las técnicas de investigación social, como son las encuestas de presupuestos temporales. Desde el dominio de tal perspectiva cuantitativa, y, por lo tanto, de la medición, se ha hecho especial hincapié en la duración: en el tiempo manifestado de duración de las distintas actividades que los sujetos realizan a lo largo de un día. Cada momento del día tiene su actividad. Cada actividad, un tiempo en clave de duración. Hay que hacer notar que tal operacionalización de los tiempos sociales no deja de ser una reducción, como ocurre en todo proceso de operacionalización (Corbetta, 2003), de ese concepto complejo que es el tiempo. Así, se hacen todos los momentos iguales, todas las duraciones idénticas, sin tener en cuenta los aspectos cualitativos de los distintos momentos y, por lo tanto, las vivencias temporales o sentido que los individuos dan a tales duraciones.

El trabajo que aquí se presenta constituye una aproximación analítica a los datos sobre el uso del tiempo que, para la Comunidad de Castilla-La Mancha, ofrece el último estudio nacional en clave de presupuestos temporales: la Encuesta de Empleo del Tiempo 2002-2003, desarrollada por el Instituto Nacional de Estadística ${ }^{2}$ Esta operación estadística está diseñada para obtener datos comparativos, a nivel de la Unión Europea, de la distribución y utilización del tiempo de las personas. Recaba información de una muestra de veinticuatro mil hogares (más de 60.000 individuos), distribuida uniformemente a lo largo del año, con el objetivo de que se encuentren representados todos los días del año a escala de estrato y comunidad autónoma, aun cuando la submuestra del denominado fin de semana (viernes a domingo) tiene un peso relativamente mayor.

La obtención de la información se realiza mediante:

a) Cuestionario del hogar, en el que se selecciona un informante. Su estructura es: la composición del hogar, atención a los menores de 10 años, disponibilidad de servicio doméstico, características y bienes de equipamiento de la vivienda principal, cultivo de plantas y cuidado de animales, ingresos económicos y ayudas recibidas por el hogar.

b) Cuestionario individual. Está especialmente centrado en las actividades del sujeto entrevistado: económicas, en el hogar, de voluntariado, ocio, etc. Salvo para el caso de la actividad laboral, el período de referencia sobre el que se cuestiona la realización de la actividad está compuesto por cuatro semanas. 
c) Diario de empleo del tiempo, durante las 24 horas de un día. Puede considerarse el registro central del estudio. En él, se pide al informante que lleve el diario a lo largo del día y lo rellene con regularidad, registrando, para cada intervalo de diez minutos, las actividades realizadas (primaria y secundaria o simultánea) y las características de la compañía con la que se realizan tales actividades

Del total de la muestra, corresponde a la Comunidad de Castilla-La Mancha una submuestra de 2.151 individuos, lo que supone el 3,6\% del total de la muestra nacional. Tamaño de la submuestra que ha de tenerse por suficiente para abordar algunas dimensiones de la realidad castellano-manchega, siempre que el proceso de desagregación no sea excesivo.

Con la finalidad de evitar niveles de demasiada desagregación, con la consiguiente pérdida de significación estadística, se ha concentrado el conjunto de actividades en seis categorías, cuya sola denominación habla de sus contenidos: cuidados personales, tiempo de trabajo, tiempo de estudios, tiempo dedicado al hogar (tareas domésticas) y el cuidado de la familia, tiempo de ocio y actividades afines y, por último, tiempo empleado en trayectos. Categorías excluyentes y que presentan muy diverso grado de homogeneidad, en cuanto al supuesto sentido del tiempo dedicado a las distintas actividades que contiene. Así, mientras tal homogeneidad es más atribuible a los tiempos de trabajo, estudio, ocio o trayectos; lo es menos con respecto a la categoría de cuidados personales o la destinada a recoger el tiempo dedicado al hogar y la familia. La primera, incluye desde actividades para la cobertura de necesidades fisiológicas (dormir, aseo), que es la denominación que adquiere esta categoría en una buena parte de los estudios de presupuestos familiares, hasta otras con una intensa proyección social, como ocurre con la comida. Por su lado, la doble cara de la categoría de actividades dedicadas al hogar y la familia, queda ya inscrita en su propia denominación.

\section{DISTRIBUCIÓN DEL TIEMPO}

A la hora de establecer la distribución del tiempo de una sociedad entre distintas actividades, es conveniente hacerlo desde un indicador como el tiempo medio social, que es el tiempo medio que dedica el conjunto de la población a cada una de las actividades, teniendo en cuenta tanto los que participan en tal actividad como los que no participan en la misma. Pues bien, la población castellanomanchega dedica once horas y veintiún minutos a cuidados personales, como dormir, asearse o comer. Al ocio y las actividades afines, lo que incluye el consumo de medios de comunicación, dedica poco más de cinco horas. Las tareas relacionadas con el hogar y el cuidado de la familia apenas superan las tres horas diarias; mientras que en trayectos se va una hora y siete minutos.

Tal vez pueda sorprender que al empleo remunerado se destinen sólo dos horas y medias diarias y al estudio apenas tres cuartos de hora. Ahora bien, ha de 
volver a subrayarse que es el tiempo destinado por el conjunto de la población castellano-manchega, por lo que, en la contabilización, también entran los no ocupados (en el trabajo) y los que no estudian, con relación a los estudios. Por ello, se muestra en la tabla 1 la tasa de participación o porcentaje de personas que realiza cada una de las actividades concretas. Así, como parece obvio, las actividades encuadradas en la categoría de cuidados personales son realizadas por la totalidad de los sujetos, como ocurre con el ocio y las actividades afines, pues algún tiempo de descanso también cabe considerar como normal, el porcentaje de población que dedica algún tiempo a los estudios se reduce al $13,6 \%$ de la población y al trabajo el $30,5 \%$. Por lo tanto, el grado en que una población participe en la actividad incide, como no podía ser de otra manera, sobre el total de tiempo medio que dedica esa población a tal actividad.

Tabla 1: Participación de la población castellano-manchega en las distintas categorías de actividades

\begin{tabular}{|l|r|}
\hline Cuidados personales & $100,0 \%$ \\
\hline Tiempo trabajo & $30,5 \%$ \\
\hline Tiempo estudios & $13,6 \%$ \\
\hline Hogar y familia & $80,0 \%$ \\
\hline Ocio y otras actividades & $98,3 \%$ \\
\hline Tiempo trayectos & $84,4 \%$ \\
\hline
\end{tabular}

Fuente: Encuesta Empleo del Tiempo, 2002-2003, INE, y elaboración propia

Por otro lado, ha de tenerse en cuenta que se trata del tiempo medio de un día cualquiera de la semana, por lo que, para su obtención, se han tenido en cuenta tanto los días laborables, como los de fin de semana. De esta manera, aun cuando exista una parte de la población que trabaje o estudie durante el fin de semana, desde la perspectiva del conjunto de la población es poco el tiempo que se dedica a tales actividades.

De hecho, como muestra la tabla 2, la distribución del tiempo de los castellano-manchegos presenta una estructura que puede considerarse bastante semejante a la que describe la distribución del conjunto de la población del estado.

Tabla 2: Tiempo medio social en Castilla-La Mancha y España (horas: minutos)

\begin{tabular}{|l|c|r|}
\hline & C. A. Castilla-La Mancha & España \\
\hline Cuidados personales & $11: 21$ & $11: 22$ \\
\hline Tiempo trabajo & $2: 30$ & $2: 39$ \\
\hline Tiempo estudios & $0: 48$ & $0: 43$ \\
\hline Hogar y familia & $3: 03$ & $2: 59$ \\
\hline Ocio y otras actividades afines & $5: 09$ & $4: 56$ \\
\hline Tiempo trayectos & $1: 07$ & $1: 10$ \\
\hline
\end{tabular}

Fuente: Encuesta Empleo del Tiempo, 2002-2003, INE, y elaboración propia 
Las principales diferencias, aun cuando han de considerarse pequeñas, se encuentran en los tiempos de trabajo y de ocio. Nueve minutos menos de tiempo medio social dedicado al trabajo retribuido en Castilla-La Mancha; a la vez que trece minutos más destinado al ocio y las actividades afines.

\section{LAS DIFERENCIAS SEGÚN CARACTERÍSTICAS DE LA LOCALIDAD}

Nos adentramos ahora, sin salir de la comunidad de Castilla-La Mancha, en lo que puede denominarse la perspectiva ecológica del uso del tiempo. Es decir, en la concreción de la distribución del tiempo en la sociedad según diferentes constituciones espaciales. Para ello, hemos dividido la muestra atribuida al conjunto de la comunidad autónoma en tres submuestras, correspondientes a tres tipos de localidades:

- Capitales de provincia -las cinco de la comunidad autónoma- y ciudades de más de 50.000 habitantes. Hábitat que puede considerarse urbano. El tamaño de esta submuestra es de 783 individuos.

- Localidades medias, cuyas poblaciones no alcancen los 50.000 habitantes, pero superen los 10.000 habitantes. El tamaño de esta submuestra es de 456 individuos.

- Localidades que tienen menos de 10.000 habitantes, coincidiendo con las que pueden ser caracterizadas como integrantes del hábitat rural. El tamaño de esta submuestra es de 912 individuos.

La distinta duración media que tienen algunas categorías de actividades a lo largo de la semana en las capitales de provincia y ciudades de más de 50.000 habitantes de la comunidad autónoma, puede observarse en la tabla 3. Como parece lógico esperar, todas las categorías experimentan alguna variación a lo largo de la semana, aun cuando lo más significativo se encuentra seguramente en que tal variación parece ir más allá de la oposición día laborable-fin de semana. Es lo que ocurre con el jueves, que aparece como el día en que los tiempos medios sociales dedicados al trabajo, los estudios y los trayectos aumentan de manera considerable; mientras que disminuye el tiempo de ocio y actividades afines. El gráfico 1 muestra ostensiblemente el cruce de los tiempos de trabajo y de ocio que se produce en el jueves. Variación que, en principio, ha de atribuirse al relativamente escaso tamaño de la submuestra que resulta de la desagregación de la submuestra por tamaño de hábitat en los siete días de la semana. Aspecto que llama a la precaución metodológica cuando se aborden, más adelante, niveles de desagregación mayor.

Tabla 3: Tiempo medio social en las capitales y ciudades de más de 50.000 habitantes en Castilla-La Mancha, según día de la semana (horas:minutos) 


\begin{tabular}{|l|r|r|r|r|r|r|}
\hline & $\begin{array}{c}\text { Cuidados } \\
\text { personales }\end{array}$ & $\begin{array}{c}\text { Tiempo } \\
\text { trabajo }\end{array}$ & \multicolumn{1}{c|}{$\begin{array}{c}\text { Tiempo } \\
\text { estudios }\end{array}$} & $\begin{array}{c}\text { Hogar y } \\
\text { familia }\end{array}$ & $\begin{array}{c}\text { Ocio y otras } \\
\text { actividades }\end{array}$ & \multicolumn{1}{c|}{$\begin{array}{c}\text { Tiempo } \\
\text { trayectos }\end{array}$} \\
\hline lunes & $11: 03$ & $2: 52$ & $0: 52$ & $3: 09$ & $5: 02$ & $0: 58$ \\
\hline martes & $10: 41$ & $2: 55$ & $1: 09$ & $3: 01$ & $5: 03$ & $1: 09$ \\
\hline miércoles & $11: 11$ & $2: 49$ & $0: 37$ & $3: 14$ & $5: 03$ & $1: 01$ \\
\hline jueves & $10: 47$ & $3: 54$ & $1: 27$ & $2: 54$ & $3: 35$ & $1: 22$ \\
\hline viernes & $11: 11$ & $2: 40$ & $0: 46$ & $3: 13$ & $5: 06$ & $1: 10$ \\
\hline sábado & $11: 28$ & $1: 29$ & $0: 25$ & $3: 03$ & $6: 16$ & $1: 18$ \\
\hline domingo & $13: 06$ & $0: 49$ & $0: 21$ & $2: 46$ & $5: 56$ & $0: 59$ \\
\hline
\end{tabular}

Fuente: Encuesta Empleo del Tiempo, 2002-2003, INE, y elaboración propia

Adquiere sentido el ligero aumento de tiempo destinado a los cuidados personales durante el sábado. Un aumento que supera las dos horas, con respecto a un día medio laborable, en el domingo. Mayor disponibilidad para tales cuidados personales, en claro detrimento del tiempo dedicado al trabajo, que queda reducido a poco más de tres cuartos de hora.

En estos espacios urbanos, el día para el ocio y actividades afines, como el consumo de medios de comunicación, es el sábado, con más de seis horas de dedicación, especialmente detraídas de los tiempos de trabajo y estudio. Por último, en este enfoque global del conjunto de la comunidad autónoma, comentar que el día que menos tiempo se dedica a las tareas relacionadas con el hogar y la familia es el domingo. En especial, se reduce el tiempo de las tareas domésticas (lavar, planchar, fregar, cocinar, etc.). Aun cuando menos que con respecto al trabajo remunerado, también se descansa el domingo del trabajo no remunerado.

\section{Gráfico 1}

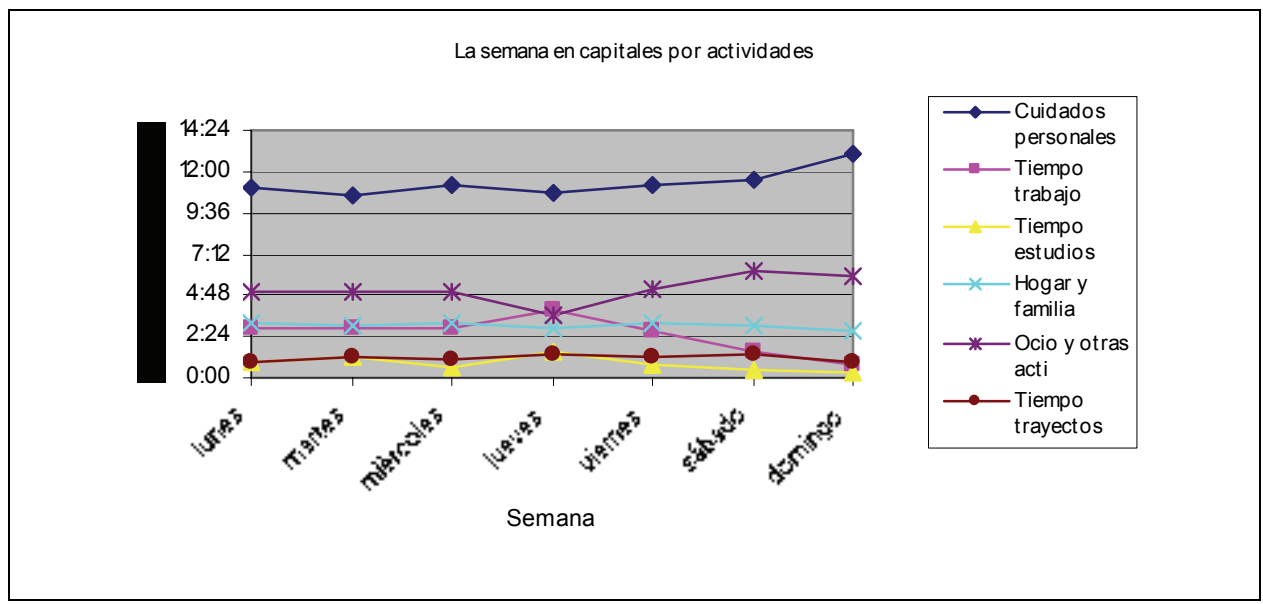

Pasando de la explicación metodológica a la sustancial, podríamos preguntarnos por la particularidad del jueves entre los residentes en los espacios 
urbanos, para que se dé tan especial uso del tiempo, en contraste con el resto de la semana: ¿preparar el fin de semana? De hecho, a partir del jueves las trayectorias de tiempo de trabajo y tiempo de ocio muestran una creciente divergencia. Podría interpretarse que el jueves es el día destinado para hacer el último gran esfuerzo en el trabajo -y el estudio- para, así, entrar de lleno en el ocio del fin de semana el día siguiente, el viernes.

Veamos ahora la distribución de la jornada, en los siete días de la semana, en las localidades medias, entre 10.000 y 50.000 habitantes (tabla 4). Destacan varios aspectos. En primer lugar, la relativamente poca diferencia entre el viernes y el sábado. En cuanto a los cuidados personales, la diferencia del tiempo social medio dedicado a ellos es de un único minuto. Ambos días se encuentran por encima del tiempo dedicado a estas tareas los otros días de la semana laboral; pero a casi dos horas del tiempo dedicado el domingo. La semejanza, aun cuando menor, se presenta también en cuanto al tiempo de trabajo. Ambos días ocupan un lugar próximo entre sí y, a la vez, a medio camino entre el resto de días laborables de la semana y el fin de semana.

Tabla 4: Tiempo medio social en localidades entre 10.000 y 50.000 habitantes en Castilla-La Mancha, según día de la semana (horas: minutos)

\begin{tabular}{|c|c|c|c|c|c|c|}
\hline & $\begin{array}{c}\text { Cuidados } \\
\text { personales }\end{array}$ & $\begin{array}{c}\text { Tiempo } \\
\text { trabajo }\end{array}$ & $\begin{array}{c}\text { Tiempo } \\
\text { estudios }\end{array}$ & $\begin{array}{c}\text { Hogar y } \\
\text { familia }\end{array}$ & $\begin{array}{c}\text { Ocio y otras } \\
\text { activ. }\end{array}$ & $\begin{array}{c}\text { Tiempo } \\
\text { trayectos }\end{array}$ \\
\hline lunes & $10: 38$ & $3: 48$ & $1: 15$ & $3: 20$ & $4: 58$ & $1: 02$ \\
\hline martes & $10: 40$ & $2: 42$ & $0: 53$ & $3: 48$ & $4: 44$ & $1: 13$ \\
\hline miércoles & $11: 05$ & $3: 45$ & $0: 44$ & $3: 12$ & $4: 18$ & $0: 56$ \\
\hline jueves & $10: 47$ & $3: 32$ & $0: 31$ & $4: 19$ & $3: 42$ & $1: 08$ \\
\hline viernes & $11: 12$ & $1: 52$ & $0: 37$ & $4: 01$ & $5: 28$ & $0: 49$ \\
\hline sábado & $11: 13$ & $1: 30$ & $0: 21$ & $3: 24$ & $6: 16$ & $1: 14$ \\
\hline domingo & $13: 01$ & $0: 45$ & $0: 16$ & $2: 44$ & $6: 13$ & $1: 00$ \\
\hline
\end{tabular}

Fuente: Encuesta Empleo del Tiempo, 2002-2003, INE, y elaboración propia

A la vista de tal distribución del uso del tiempo durante la semana en estas localidades medias, puede decirse que el viernes es algo más que la puerta al fin de semana. Prácticamente, forma parte del fin de semana. La mayor diferencia con respecto al sábado se encuentra en el tiempo de ocio y actividades afines, donde nos volvemos a encontrar al sábado como el día de mayor dedicación a esta categoría de actividades. Los tres cuartos de hora que distancian la duración del tiempo de ocio del viernes, de la del sábado, se proyectan principalmente en el tiempo dedicado a las tareas del hogar y la familia. Es decir, el viernes se dedican aún más de treinta y cinco minutos por encima del sábado a las tareas relacionadas con el hogar y la familia.

Como puede verse en el gráfico 2, el jueves se produce una ostensible aproximación entre las trayectorias de las categorías tiempo de trabajo y tiempo de 
ocio, principalmente, y un cruce con el tiempo dedicado a las tareas domésticas y de cuidado familiar. En cualquier caso, si se tiene en cuenta el gráfico 1, llama la atención la mayor variación del tiempo destinado a estas tareas domésticas a lo largo de la semana y que ya no se produce cruce entre la trayectoria de tiempo de trabajo y tiempo de ocio. Tan solo se da una aproximación; pero siempre con el tiempo de actividades ociosas por encima.

\section{Gráfico 2}

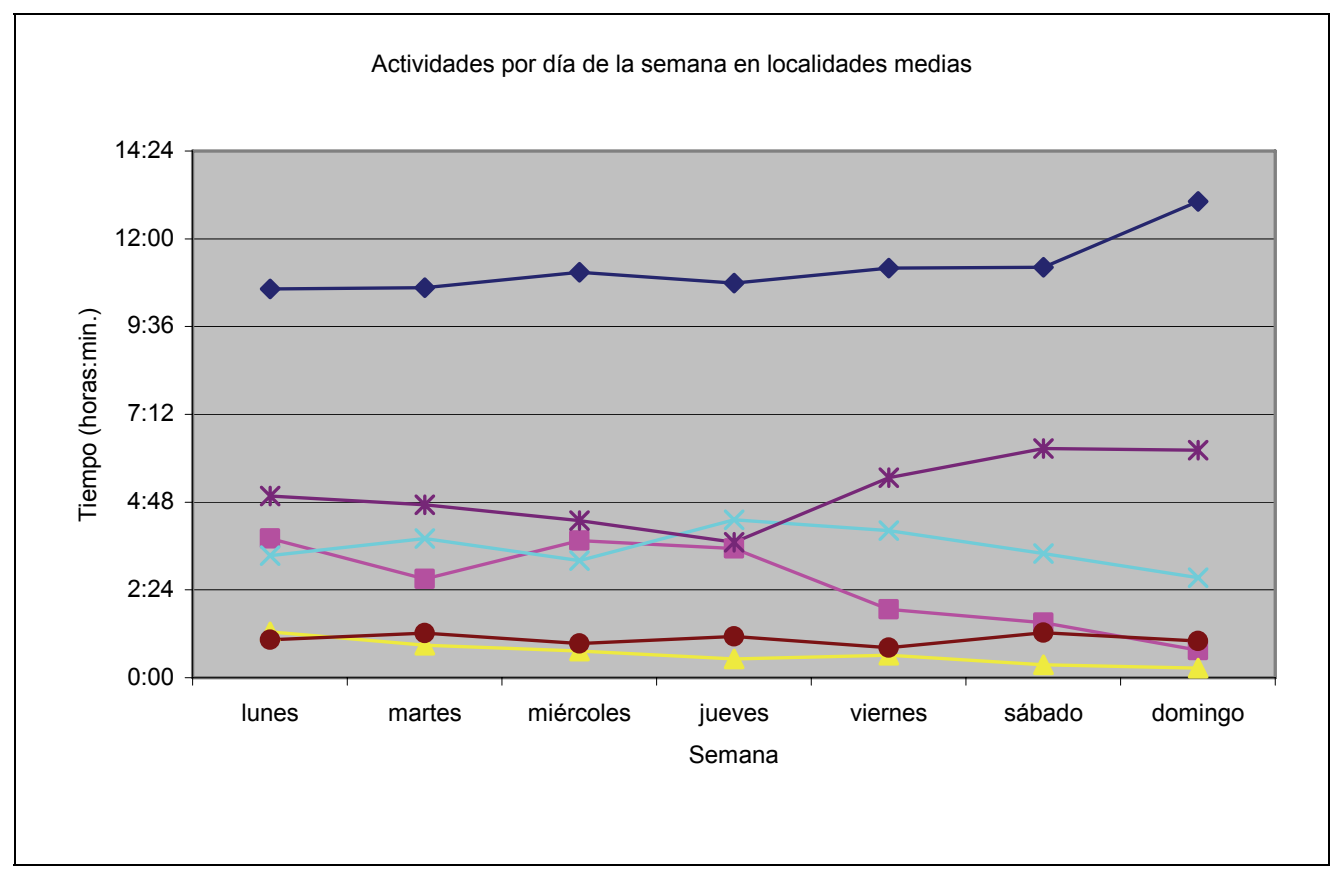

Tal cruce entre las trayectorias de los tiempos de trabajo y de ocio tampoco se produce en las localidades rurales, de menos de 10.000 habitantes (gráfico 3). Durante toda la semana, el tiempo que se dedica al ocio y actividades afines es superior al tiempo de trabajo. Pero la aproximación entre ambas trayectorias se produce aquí el martes, en lugar del jueves. Parece que puede hablarse de dos tipos de semana, una en los hábitat urbano y semiurbano, con una mayor actividad laboral los jueves y una plena entrada en el fin de semana los viernes, que hacen de este día muy similar al sábado; y una semana en las localidades rurales, con más diferencia entre el viernes y el sábado, de manera que el primero se constituye en un día laborable más. Por otro lado, el tiempo dedicado a las tareas domésticas y los cuidados familiares aparece más homogéneo, a lo largo de la semana, que en las localidades medias. 
Gráfico 3

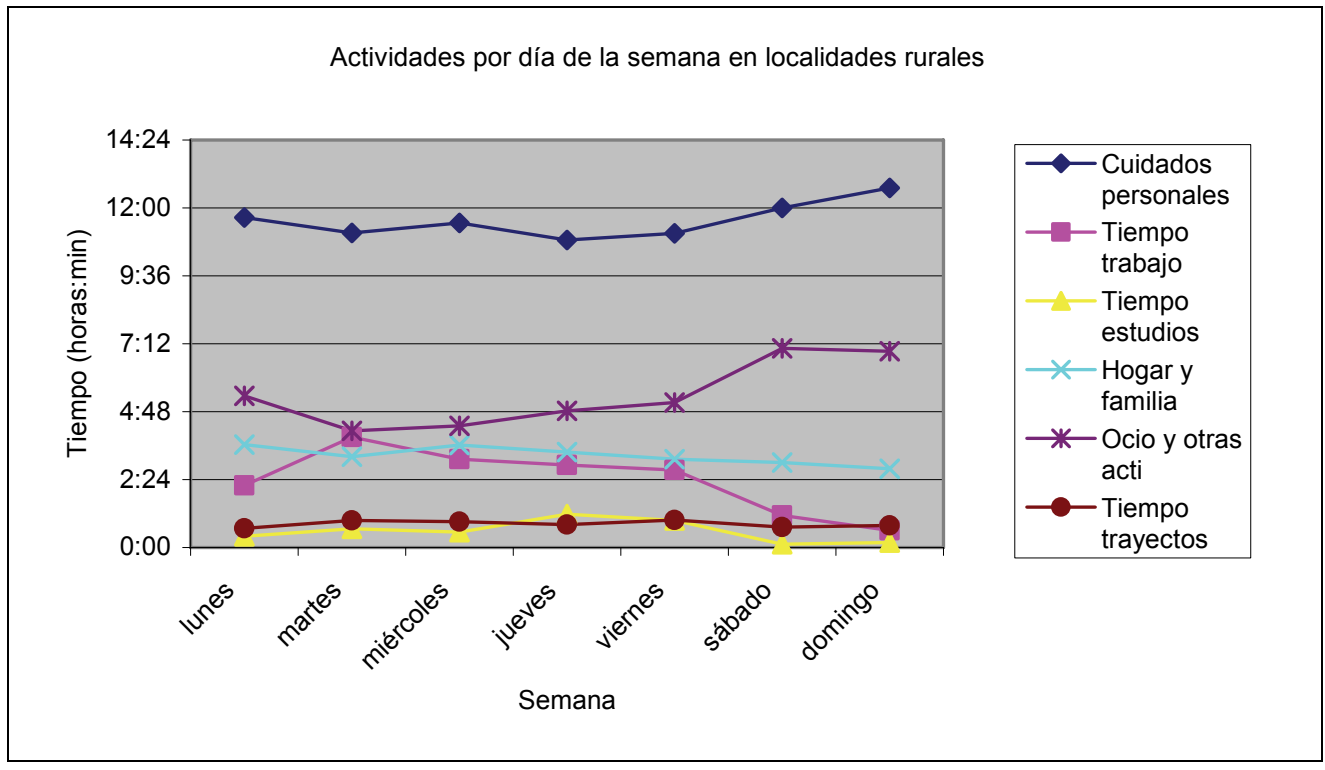

Comparemos ahora directamente las trayectorias a lo largo de la semana de las distintas categorías de actividades en los tres hábitats. Así se produce una desigual semana entre los tres hábitat si se tiene en cuenta el tiempo de trabajo (gráfico 4):

- En los tres hábitat, baja el viernes el tiempo medio dedicado al trabajo, pero bastante más en las localidades medias, descendiendo menos en el hábitat rural.

- El aumento del tiempo medio de trabajo en capitales los jueves y los martes en el hábitat rural. En las localidades medias, el aumento se produce el miércoles.

- La semana empieza con una distinta duración del tiempo de trabajo según el hábitat. Dedicación social media relativamente alta en las localidades medias y relativamente baja en las localidades rurales. Las capitales empiezan la semana con un tiempo social de dedicación al trabajo entre los dos otros tipos de localidad.

- Tendencia a la convergencia durante el fin de semana. La caída desde el jueves hasta el domingo es más pronunciada en las capitales y ciudades de más de 50.000 habitantes. 


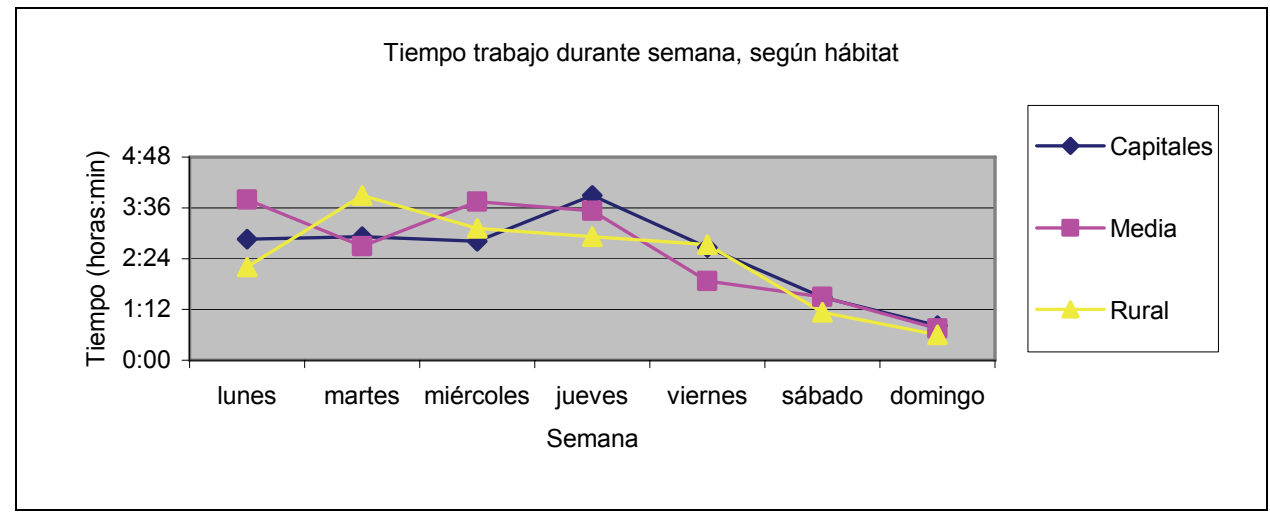

Gráfico 4

A la hora de comparar el tiempo dedicado a las tareas del hogar y los cuidados familiares, destaca (gráfico 5) que, salvo los miércoles, la dedicación es constantemente superior en las localidades medias. La diferencia de este tipo de hábitat marca su máxima distancia los jueves. Sin embargo, hay una convergencia en el domingo. Puede considerarse una especie de convergencia en mínimos. Como si las dos horas y tres cuartos que marca significase una especie de mínimo de tiempo que la sociedad, encarnada en sus individuos, ha de dedicar a tales tareas.

En cualquier caso, este tiempo parece más estable en los hábitat urbano y rural, que en las localidades medias, presentándose aquí importantes oscilaciones a lo largo de la semana.

\section{Gráfico 5}

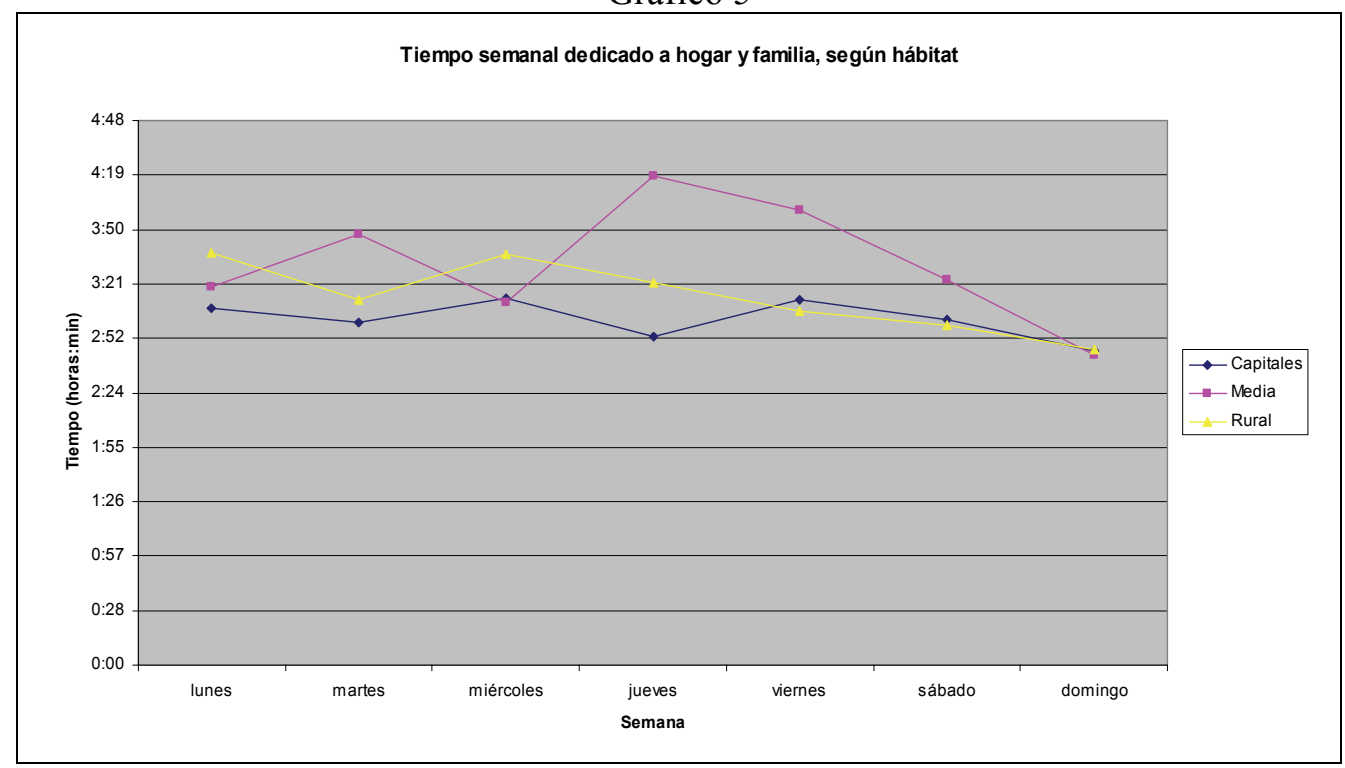


La proporción de la jornada destinada al ocio y actividades afines experimenta notables variaciones a lo largo de la semana en los tres tipos de localidad; pero es relativamente más estable en las localidades rurales (gráfico 6). En la trayectoria semanal de esta categoría de actividad en los otros dos tipos de localidad sobresale el notable descenso del jueves, que, como se ha visto, presenta un aumento de la actividad laboral.

En los tres tipos de localidad, el sábado es el día que dedica más tiempo proyectado al ocio. En cualquier caso, durante el fin de semana, las localidades rurales usan mayor parte de la jornada para este tipo de actividades; mientras que durante la semana laboral, con la excepción del referido jueves, tiende a ser menor el total de tiempo de ocio en estas localidades rurales. No obstante, el lunes y el viernes la proporción de la jornada dedicada al ocio es bastante similar en las tres, cuestión que nos devuelve a la reflexión sobre el papel del viernes. Más parecido al sábado en las localidades urbanas y semiurbanas; pero no tanto porque se dedique más tiempo al ocio el viernes, sino por la relativamente menor dedicación a tal actividad los sábados en estas localidades. De esta manera, el salto desde el viernes al sábado es mayor en el ámbito rural.

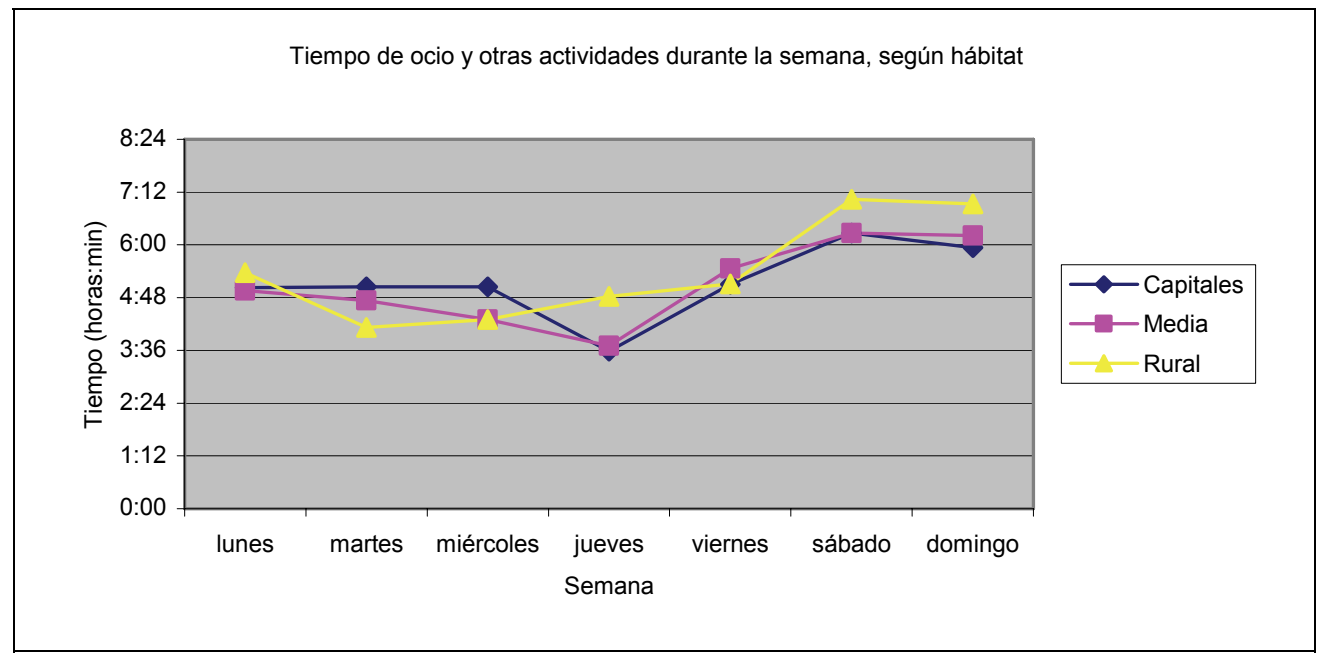

Gráfico 6

¿Dónde se encuentra, entonces, la razón del gran salto entre el viernes y el sábado en las localidades rurales y la falta del mismo en las localidades urbanas y semiurbanas, que nos lleva a incluir este día en el fin de semana? De las categorías de actividades que nos queda por comparar directamente, el tiempo social medio destinado a los estudios o los estudios varía de un tipo de localidad a otro; pero ambas categorías de actividades tienen un peso relativamente bajo en la 
estructuración de la jornada social media, bien es cierto que menor, en ambos casos, en las localidades rurales. Habrá que buscar la diferencia en las trayectorias semanales del tiempo dedicado a los cuidados personales.

Como puede verse en la tabla 5 y su correspondiente reflejo en el gráfico 7 , el salto importante entre el viernes y el sábado en las localidades rurales proviene del tiempo dedicado a los cuidados personas. Mientras en las localidades urbanas (capitales y ciudades de más de 50.000 habitantes) y medias (entre 10.000 y 50.000 habitantes) las diferencias en el tiempo destinado a cuidados personales entre viernes y sábado es de algunos minutos, no sobrepasando los dieciséis, en el caso de las localidades rurales la diferencia se aproxima a la hora. Por el contrario, la diferencia entre el jueves y el viernes en el tiempo dedicado a los cuidados personales supera los veinte minutos en las localidades de mayor tamaño, mientras que se queda en los ocho minutos en el caso de las localidades rurales.

Tabla 5: Tiempo medio social dedicado a cuidados personales, según tipo de localidad y día de la semana (horas:minutos)

\begin{tabular}{|l|r|r|r|}
\hline & Capitales & Media & Rural \\
\hline lunes & $11: 03$ & $10: 38$ & $11: 40$ \\
\hline martes & $10: 41$ & $10: 40$ & $11: 07$ \\
\hline miércoles & $11: 11$ & $11: 05$ & $11: 28$ \\
\hline jueves & $10: 47$ & $10: 47$ & $10: 52$ \\
\hline viernes & $11: 11$ & $11: 12$ & $11: 06$ \\
\hline sábado & $11: 28$ & $11: 13$ & $12: 00$ \\
\hline domingo & $13: 06$ & $13: 01$ & $12: 42$ \\
\hline
\end{tabular}

Fuente: Encuesta Empleo del Tiempo, 2002-2003, INE, y elaboración propia Gráfico 7

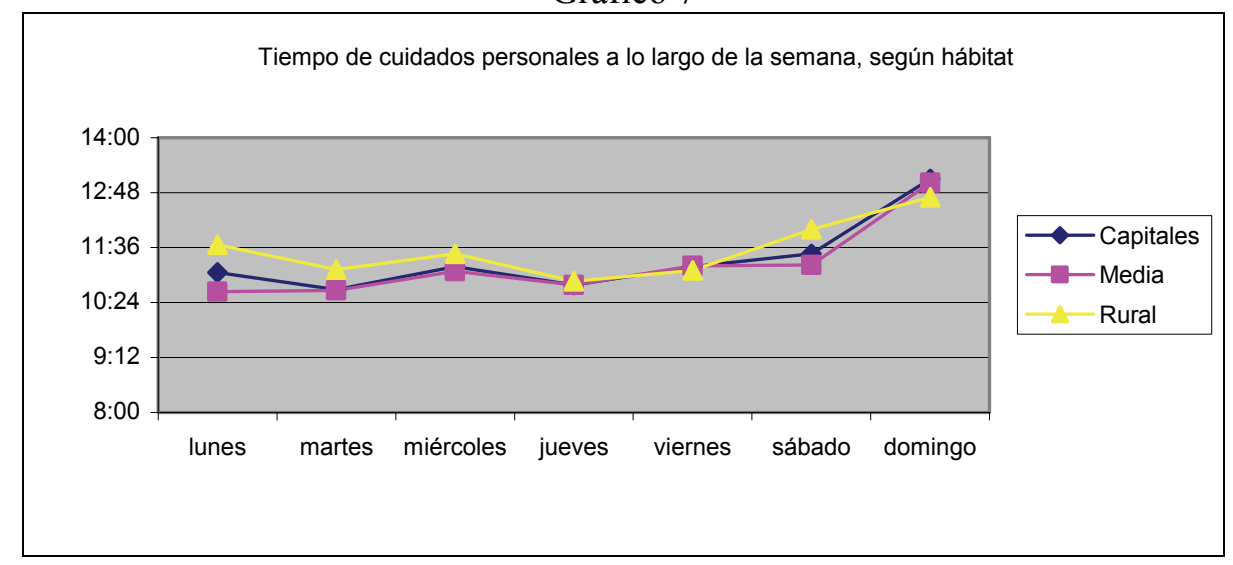


Parece poder hablarse así de dos tipos de semana en la comunidad de Castilla-La Mancha, según se haga la diferencia entre semana laboral y fin de semana, que tiene como uno de los pivotes centrales el tiempo dedicado a los cuidados personales, que puede erigirse en un indicador de descanso: en la medida que mayor proporción de la jornada se dedica a los cuidados personales, mayor posibilidad de descanso hay. Así, se puede erigir el jueves en punto de partido para todas las localidades, dedicándose un tiempo muy similar para los cuidados personales, al descanso. Sin embargo, durante el viernes y el sábado este tiempo de descanso aumenta de forma gradual en las localidades urbanas, para dar un salto más que notable, hasta situarse por encima de las trece horas, en el domingo, siendo el día con mayor proporción de jornada dedicada a los cuidados personales en todas las localidades. Pero la forma de alcanzar el domingo es distinta en las localidades rurales: la diferencia principal se da entre el viernes y el sábado, siendo menor la del domingo; mientras que es relativamente pequeña la que va desde el jueves al viernes. Por eso, puede decirse que el viernes aparece más inscrito en la semana laboral, que en el fin de semana, en las localidades rurales; pero, a su vez, también puede señalarse que el sábado en las localidades urbanas está lleno de ocupaciones (trabajo remunerado y tareas domésticas) que lo separan significativamente del domingo.

\section{EL SENTIMIENTO DE AGOBIO}

Aun cuando la observación de la distribución del tiempo a lo largo de las jornadas es el objetivo central y protagonista de la Encuesta sobre Usos del Tiempo, también permite una aproximación a aspectos que pueden considerarse más cualitativos, aun cuando dentro de la perspectiva cuantitativa. Acercamientos a la forma general de vivir el tiempo, a cierta intensidad del tiempo. Esto lo hace sobre todo a partir de las preguntas sobre el sentimiento de agobio. Conectemos con las diferencias al respecto según el tipo de localidad, en un contexto general de la comunidad (tabla 6) marcado por un relativamente escaso sentimiento de agobio, ya que casi la mitad de sus habitantes, el 49,2\%, expresa que casi nunca experimenta tal sentimiento. Sólo el $11,1 \%$ de los adultos de la comunidad de Castilla-La Mancha dice sentirlo muy frecuentemente.

En principio, se aprecia un menor nivel de agobio en el hábitat rural. Parece que se mantiene el estereotipo cuando se abordan los datos desde el conjunto del colectivo; pero no pasa lo mismo cuando se entra en el uso del tiempo de las distintas categorías sociales.

Tabla 6: Sentimiento agobio, según tipo hábitat. Porcentaje de tipo hábitat 


\begin{tabular}{|l|l|r|r|r|r|}
\hline \multicolumn{2}{|c|}{} & \multicolumn{3}{|c|}{ Tipo hábitat } & \multicolumn{1}{c|}{ Total } \\
\hline \multicolumn{2}{|l|}{} & Capitales & \multicolumn{1}{c|}{ Medias } & Rural & \\
\hline $\begin{array}{l}\text { Sentimiento } \\
\text { agobio }\end{array}$ & $\begin{array}{l}\text { Muy } \\
\text { frecuentemente }\end{array}$ & $9,5 \%$ & $12,8 \%$ & $11,6 \%$ & $11,1 \%$ \\
\hline & Algunas veces & $44,0 \%$ & $50,0 \%$ & $31,1 \%$ & $39,7 \%$ \\
\hline & Casi nunca & $46,4 \%$ & $36,9 \%$ & $57,3 \%$ & $49,2 \%$ \\
\hline Total & $100,0 \%$ & $100,0 \%$ & $100,0 \%$ & $100,0 \%$ \\
\hline
\end{tabular}

Fuente: Encuesta Empleo del Tiempo, 2002-2003, INE, y elaboración propia

A pesar de que los habitantes en localidades rurales presentan menor sentimiento de agobio, no puede hablarse de una relación directa entre tamaño del hábitat y extensión de este sentimiento de agobio. De hecho, según la distribución que muestra la tabla 6 , las localidades con mayor sentimiento de agobio son las medias, entre diez mil y cincuenta mil habitantes.

Sin embargo, las cosas cambian cuando se profundiza y se compara la sensación de agobio entre los sexos.

Tabla 7: Sentimiento agobio, según tipo hábitat y género

Porcentaje de tipo hábitat, dentro de cada género

\begin{tabular}{|l|l|r|r|r|r|r|r|}
\hline \multicolumn{2}{|c|}{} & \multicolumn{6}{|c|}{ Sexo } \\
\hline & \multicolumn{3}{|c|}{ varones } & \multicolumn{3}{c|}{ mujeres } \\
\hline & \multicolumn{3}{|c|}{ Tipo hábitat } & \multicolumn{3}{c|}{ Tipo hábitat } \\
\hline \multicolumn{2}{|l|}{} & Capitales & Medias & Rural & Capitales & Medias & Rural \\
\hline $\begin{array}{l}\text { Sentimiento } \\
\text { agobio }\end{array}$ & $\begin{array}{l}\text { Muy } \\
\text { frecuentemente }\end{array}$ & $6,2 \%$ & $6,2 \%$ & $8,9 \%$ & $12,2 \%$ & $19,0 \%$ & $13,9 \%$ \\
\hline & Algunas veces & $41,5 \%$ & $46,9 \%$ & $21,8 \%$ & $46,1 \%$ & $52,9 \%$ & $39,2 \%$ \\
\hline & Casi nunca & $52,3 \%$ & $46,9 \%$ & $69,3 \%$ & $41,7 \%$ & $27,6 \%$ & $46,9 \%$ \\
\hline
\end{tabular}

Fuente: Encuesta Empleo del Tiempo, 2002-2003, INE, y elaboración propia

Entonces, nos encontramos con la polaridad existente entre los varones de localidades rurales, pues mientras presentan la mayor proporción de ausencia de sentimiento de agobio (69,3\% no lo experimenta casi nunca), el $8,9 \%$ lo experimenta muy frecuentemente, presentando la mayor proporción entre los varones. Pero, sobre todo, sobresale que las mayores proporciones de experiencia habitual del sentimiento de agobio se encuentran entre mujeres, destacando especialmente la de las mujeres de localidades medias, donde casi una de cada cinco mujeres ha manifestado tener sentimiento de agobio muy frecuentemente. 
Situación que seguramente hay que interpretarla a la luz de la comparación que, entre los tres tipos de localidades, se presenta en el gráfico 2, de manera que las localidades medias son las que más tiempo dedican a las tareas del hogar y a los cuidados familiares. Tiempos y trabajos que se concentran especialmente entre las mujeres. Algo que parece conducirnos, poco a poco, al tipo de actividad que principalmente se realiza y que constituye la principal fuente de integración social y de referencia de posición en la estructura social. Es decir, a la idea de que es el tipo de actividad y, por supuesto, el tiempo dedicado a una actividad en cuanto tal vez se encuentre socialmente poco valorado, lo que puede estar en la base del sentimiento de agobio.

Observando la tabla 8 con las precauciones metodológicas que derivan de un nivel de desagregación mayor y, por lo tanto, de submuestras de tamaño cada vez menor, se percibe que la mayor intensidad del sentimiento de agobio se encuentra entre las amas de casa de las localidades medias, seguidas de las viudas de las localidades rurales y de las propias localidades medias. Sin embargo, los jubilados, especialmente de las localidades mayores (capitales y ciudades de más de 50.000 habitantes) y de las localidades rurales los que menos sentimiento de agobio experimentan. Puede decirse que prácticamente desconocen este sentimiento, ya que casi cuatro de cada cinco no lo experimentan casi nunca.

Tabla 8: Sentimiento de agobio según tipo hábitat y ocupación

Porcentaje de cada tipo de hábitat, dentro de cada ocupación

\begin{tabular}{|c|c|c|c|c|}
\hline \multirow{2}{*}{\multicolumn{2}{|c|}{ Ocupación }} & \multicolumn{3}{|c|}{ Sentimiento agobio } \\
\hline & & Muy frecuentemente & Algunas veces & Casi \\
\hline $\begin{array}{l}\text { Ocupado t. } \\
\text { completo }\end{array}$ & Capitales & $11,3 \%$ & $51,8 \%$ & $36,9 \%$ \\
\hline & Medias & $5,8 \%$ & $57,2 \%$ & $37,0 \%$ \\
\hline & Rural & $9,8 \%$ & $31,5 \%$ & $58,7 \%$ \\
\hline Parado & Capitales & $9,3 \%$ & $32,6 \%$ & $58,1 \%$ \\
\hline & Medias & $6,3 \%$ & $25,0 \%$ & $68,8 \%$ \\
\hline & Rural & $11,1 \%$ & $37,0 \%$ & $51,9 \%$ \\
\hline Estudiante & Capitales & $3,7 \%$ & $55,6 \%$ & $40,7 \%$ \\
\hline & Medias & $9,5 \%$ & $33,3 \%$ & $57,1 \%$ \\
\hline & Rural & $3,1 \%$ & $40,6 \%$ & $56,3 \%$ \\
\hline Jubilación & Capitales & , $0 \%$ & $20,3 \%$ & $79,7 \%$ \\
\hline & Medias & $12,8 \%$ & $36,2 \%$ & $51,1 \%$ \\
\hline & Rural & $7,7 \%$ & $14,4 \%$ & $77,9 \%$ \\
\hline Pensión viudedad & Capitales & $8,0 \%$ & $36,0 \%$ & $56,0 \%$ \\
\hline & Medias & $20,0 \%$ & $65,0 \%$ & $15,0 \%$ \\
\hline & Rural & $23,8 \%$ & $26,2 \%$ & $50,0 \%$ \\
\hline Ama de casa & Capitales & $11,8 \%$ & $35,5 \%$ & $52,7 \%$ \\
\hline & Medias & $24,7 \%$ & $54,1 \%$ & $20,0 \%$ \\
\hline & Rural & $13,0 \%$ & $39,1 \%$ & $47,8 \%$ \\
\hline
\end{tabular}

Fuente: Encuesta Empleo del Tiempo, 2002-2003, INE, y elaboración propia 
Las viudas y las amas de casa aparecen entre las categorías que experimentan una mayor sensación de agobio, especialmente en las localidades medias y rurales. Entre los ocupados a tiempo completo, resaltar que se trabaja con menos agobio de tiempo en el hábitat rural. Sin embargo, hay que resaltar que es más agobiante el paro en hábitat rural. En las localidades medias parece menos agobiante, desde la perspectiva del uso del tiempo, la situación de paro. Resultados que nos indican la importancia del hábitat, del tipo de localidad, a la hora de la experimentación del sentimiento de agobio; pero, sobre todo, la relevancia del lugar en la estructura socio-ocupacional dentro de cada uno de los hábitat, llevándose la peor parte las mujeres.

\section{CONCLUSIONES}

El trabajo sólo ha pretendido una presentación de algunos aspectos de la distribución del tiempo en el conjunto de la comunidad autónoma de Castilla-La Mancha. Puede hablarse de una panorámica de conjunto, a la luz de los resultados ofrecidos por la Encuesta de Empleo del Tiempo desarrollada por el Instituto Nacional de Estadística para España, bajo la coordinación de Eurostat.

Debido precisamente a que está atravesado por lo social, ha de tenerse en cuenta que el uso del tiempo tiende a variar de forma importante entre las distintas categorías de la sociedad: género, edad, ocupación, etc. Por ello, lo aquí ofrecido tiene el significado de un primer paso, que ha dado especial relevancia al hábitat o tamaño de la localidad.

El análisis de la distribución del uso del tiempo a lo largo de la semana, teniendo en cuenta tres tipos de localidad (capitales y ciudades grandes, localidades medias y localidades rurales), nos ha dado dos tipos de semana, especialmente a partir del lugar que ocupa el viernes. Tendencia a incluirlo en el fin de semana en las localidades urbanas y semiurbanas; a excluirlo en el hábitat rural.

Mientras que en las localidades rurales aparece una clara diferencia entre la semana laboral y un fin de semana más homogéneo, formado por el sábado y el domingo dedicados al descanso, en las localidades urbanas se configura una especie de tripartición de la semana. Por un lado, la semana plenamente laboral de lunes a jueves, siendo este día el que más tiempo dedica al trabajo remunerado, pareciendo significar un esfuerzo por acabar con las tareas y no dejar nada pendiente. Por otro lado, una forma de fin de semana leve, constituido por el viernes y el sábado, donde el tiempo de ocupaciones (laborales, estudios, tareas domésticas) se reduce; pero queda una proporción importante, de manera que el tiempo social medio de descanso o cuidados personales se ve frenado. Por último, un domingo dedicado en su mayor parte, por encima de las trece horas, al descanso.

Por otro lado, el sentimiento de agobio ha tomado las características de un sentimiento de integración. Con respecto al mismo y teniendo el carácter central 
asumido del tipo de localidad, hay que resaltar que la principal oposición se ha establecido entre localidades medias, con un mayor sentimiento de agobio, y las otras localidades. No obstante, al tener en cuenta otras categorías, como el género o la ocupación, lo que destaca es el mayor sentimiento de agobio de las mujeres en general, especialmente de las amas de casa y las viudas, y de las mujeres en localidades medias en particular.

Por último, destacar que esta aproximación al uso del tiempo en Castilla-La Mancha puede tener su proyección en el conjunto del territorio español. En especial, la observación de la existencia de una semana partida en tres tal vez pueda ayudar a resolver el constante problema metodológico del lugar que ocupa el viernes en los estudios de presupuestos temporales, sin saber si situarlo entre los días laborales (como se hace ahora) o dentro del fin de semana. Podría hablarse así de: semana laboral (lunes-jueves), inmersión en el fin de semana (viernes y sábado) y domingo.

\section{NOTAS}

${ }^{1}$ Un horizonte institucionalizado en asociaciones como la International Association for Time Use Research (IATUR) o la European Foundation for the Improvement of Living and Working Conditions

${ }^{2}$ Los microdatos del estudio pueden obtenerse en las páginas web del INE: www.ine.es

\section{BIBLIOGRAFÍA}

CORBETTA, P. (2003): Metodología y técnicas de investigación social, Madrid, McGrawHill

SZALAI, A. (ed.) (1972): The use of time, The Hague, Mouton. 